\title{
Multiple partners are associated with higher testosterone in North American men and women
}

\author{
Sari M. van Anders *, Lisa Dawn Hamilton, Neil V. Watson \\ Department of Psychology, Simon Fraser University, 8888 University Drive, Burnaby BC, Canada V5A 1 S6
}

Received 4 December 2006; revised 4 January 2007; accepted 6 January 2007

Available online 27 January 2007

\begin{abstract}
Previous research has shown that being partnered is associated with lower testosterone $(\mathrm{T})$ in men and women. To address how multiple partners may be associated with T, we examined 47 men and 48 women who were single, monoamorously partnered (partnered), polyamorous (having multiple committed relationships), or in a polyamorous lifestyle but not currently multipartnered. Men who were partnered had lower T than all other men, and polyamorous men had higher T than single men. Polyamorous women had higher T than all other women. Measures of sociosexual orientation (SOI) and sexual desire differed in women by relationship type, but not in men. Findings are interpreted in light of 'competitive' and 'bond-maintenance' relationship orientations and statuses.
\end{abstract}

(C) 2007 Elsevier Inc. All rights reserved.

Keywords: Androgen; Affiliation; Gender; Sex; Pair bond; Sexual desire; Sociosexual orientation; Relationships; Testosterone

\section{Introduction}

Associations between testosterone ( $\mathrm{T}$ ) and partnering have received increasing attention since original reports showing higher $\mathrm{T}$ in single than married men (Booth and Dabbs, 1993; Mazur and Michalek, 1998). Further studies have replicated and extended these findings such that single men have been shown to have higher $\mathrm{T}$ than partnered men, whether partnered status is married, common-law, or long-term relationships (e.g. Gray et al., 2002; Burnham et al., 2003; Gray et al., 2004a,b). In addition, studies suggest that this effect occurs in heterosexual but not non-heterosexual men and may be more apparent in non-heterosexual women (van Anders and Watson, 2006a). The majority of studies have been carried out with North American populations, and studies with Asian populations tend to find weaker but somewhat consistent findings (e.g. Sakaguchi et al., 2006). One study with a population (i.e. Kenyan Swahili men) where monoamorous relationships like marriage are not the only long-term relationship possibility found that polygynously

\footnotetext{
* Corresponding author. Fax: +1 6042913427.

E-mail address: saria@sfu.ca (S.M. van Anders).
}

married men had higher $\mathrm{T}$ than monogamously married men (Gray, 2003).

Of course, monoamorous relationships are not the only longterm relationship possibility in North American cultures either, and one additional relationship type is polyamory. Polyamory is not as widely known as other relationship types, and though there are various definitions, it generally refers to the "... philosophy and practice of loving multiple people simultaneously" (Introduction to Polyamory). Polyamory is a specific approach to relationships that includes nonpossessiveness, honesty, and openness (and thus differs from 'cheating' or adultery). Polyamory differs from North American polygyny in many ways; e.g. it is not regulated through religious strictures and/or communities, it is not focused on multiple marriages between one man and many women, and it is associated with values related to freedom to pursue emotional and sexual intimacy and expression. Polyamory also differs from swinging or open relationships (though not all agree on this distinction), especially with polyamory's focus on love (poly amor=many loves) and emotional connections in multiple romantic/sexual relationships.

It has been posited that being in a monoamorous committed relationship might be associated with lower $\mathrm{T}$ because this is a 
bond-maintenance context in the testosterone trade-off framework (van Anders and Watson, 2006b). In this framework, low $\mathrm{T}$ is associated with bond-maintenance behaviors, contexts, or orientations (aimed at promoting intimate caring bonds with others), and high $\mathrm{T}$ is associated with competitive behaviors, contexts, or orientations (aimed at acquiring or defending resources, including partners and offspring). If looking for additional partners, or the possibility of additional partners, is a competitive situation, then polyamory might be associated with higher $\mathrm{T}$. Though people in monoamorous relationships often seek sexual/romantic encounters beyond their relationship, on average this should occur less frequently than in individuals with a lifestyle approach that makes this possibility explicit. And, people in polyamorous or monoamorous relationships may not differ in bond-maintenance orientation or behaviors because they may be similarly bond-maintenance oriented (i.e. in long-term relationships).

Thus, we hypothesized that polyamorous individuals might exhibit higher $\mathrm{T}$ than monoamorously partnered individuals. We also hypothesized that polyamorous individuals might exhibit higher $\mathrm{T}$ than single individuals because their lifestyle is explicitly oriented toward the possibility and likelihood of new partners in a way that being single is not. An interesting alternative possibility is that polyamorous individuals might exhibit lower $T$ than single individuals because they are engaged in more bond-maintenance behaviors or are more bond-maintenance-oriented and exhibit similar $\mathrm{T}$ levels to monoamorously partnered individuals because these groups may be similarly oriented towards long-term relationships.

We were interested in whether sex moderated the association between $\mathrm{T}$ and relationship type because previous research with women has resulted in a complex pattern of results (van Anders and Watson, 2006a, 2007). Also, we were interested in how SOI scores (sociosexual orientation inventory; Simpson and Gangestad, 1991) might be associated with the T-partnering association since SOI is a measure of self-reported willingness to engage in sexual activity outside of committed, emotional contexts. It is therefore unclear whether monoamorously partnered individuals should differ from polyamorous individuals in SOI scores; should they be less restricted (i.e. more willing), more restricted (i.e. less willing), or similar because of polyamory's focus on multiple but committed relationships? Similarly, we were interested in how sexual desire might be associated with relationship status.

\section{Methods}

\section{Participants}

Participants were recruited through advertisements in the community or through listservs (e-mail discussion groups) and received small reimbursements for their involvement and time. Participants self-identified their sex, and we had 59 women, 47 men, two male-to-female trans-identified individuals, and one bigendered identified individual. Participants who were using medications that affected gonadal steroids (including hormonal contraceptives) were excluded from the study, leaving 48 women (mean age $=31.48$ years; $\min =18$ years, $\max =60$ years) and 47 men (mean age $=31.98$ years, $\min =19$ years, $\max =54$ years). Participants were diverse in their ethnicity, occupation, and education, though all participants but two were high school graduates.
For sexual orientation, participants self-identified and also responded to the Kinsey questions of sex-directed fantasy and behavior (Kinsey et al., 1948). From the responses to the Kinsey questions, we divided our participants into heterosexual (exclusive or near exclusive opposite-sex fantasy and behavior) and non-heterosexual (some to exclusive same-sex fantasy or behavior). This resulted in 36 heterosexual men, 10 non-heterosexual men, 26 heterosexual women, and 22 non-heterosexual women. One man did not respond to the Kinsey questions. Because of a close match between sexual orientation by selfidentification and the Kinsey questions, and because we have used the Kinsey questions in the past (e.g. van Anders and Watson, 2006a, 2007), we used sexual orientation as per the Kinsey questions in our analyses.

We recruited participants who were single or in relationships, but two dating participants (one man and one woman) volunteered for the study and were excluded from subsequent analyses because we were not testing this group. Participants indicated their relationship status and their number of partners, and we grouped individuals into single (11 men; 13 women), partnered (11 men; 6 women), polyamorous currently with multiple partners ( $12 \mathrm{men}$; 17 women), and polyamorous lifestyle (poly lifestyle) but not currently with multiple partners (6 men; 4 women). We grouped individuals by the following criteria. Single participants identified themselves as 'single' and responded to questions for people with no current partners. Partnered people responded to questions for people with one current partner and identified as being in a long-term relationship.

Polyamorous individuals used various terms to identify that they were in relationships that were or were not exclusive, with or without one primary partner, serious or casual, etc. Polyamorous participants were mostly (but not exclusively) recruited from polyamory groups and responded to questions for people with two or more current partners. There was one participant we included in this category who responded to questions for people with one partner but identified as 'married and dating' and was recruited through the polyamory groups. Poly lifestyle participants responded to questions for people with no or one current partner, but we categorized them as poly lifestyle instead of single, dating, or partnered because they either were recruited through poly networks or groups and/or identified their relationship status using words that indicated they were not currently having multiple committed relationships.

The plurality of polyamorous women had two partners $(n=6)$, followed by three partners $(n=5)$, four partners $(n=3)$, and one each for five and six partners (with three non-responders). The majority of polyamorous men also had two partners $(n=6)$, followed by four partners $(n=3)$, and then one each for three and five partners (with five non-responders). The majority of polyamorous participants reported having a primary partner (10 of 16 women, 8 of 11 men). All women reported that all their partners were aware of them having multiple partners, as did all men but one.

\section{Materials and procedure}

This study was subject to prior approval by the SFU Research Ethics Board, and all participants completed informed consent forms. Participants were tested mainly between 1300 and $2100 \mathrm{~h}$ to control for diurnal rhythms in T, except six who were tested outside this timeframe because of methodological constraints (we controlled for time of sampling in our analyses). Participants were tested in the lab, in their homes, or at public testing sites. Many of the polyamorous participants were tested at one of two meetings of regional polyamory groups (we controlled for month of sampling in our analyses because of this and seasonal patterns in $\mathrm{T}$ : van Anders et al., 2006). At one of these groups, participants were given an abbreviated version of the questionnaire because of time constraints. Participants provided a saliva sample and completed a brief questionnaire about their demographics, health and background, and relationship status.

This questionnaire included the Sociosexual Orientation Index (SOI; Simpson and Gangestad, 1991) and the Sexual Desire Inventory (SDI; Spector et al., 1996) because both constructs may be associated with relationship type. The SDI provides three scales: solitary SDI, dyadic SDI, and total SDI. We collected information about menstrual cycle status but did not control for menstrual phase. Though studies have shown that variables related to partnering or sexuality can shift over the menstrual cycle (e.g. Gangestad et al., 2004; Jones et al., 2005), research has found that menstrual phase does not need to be controlled unless cycle phase is of interest (Dabbs and de la Rue, 1990). 
Saliva samples were collected in polystyrene tubes pretreated with sodium azide, and frozen after collection at $-20{ }^{\circ} \mathrm{C}$ until assay. Saliva was stimulated with the use of an inert gum (Trident cherry sugar-free). The samples were assayed for testosterone using radioimmunoassay in one batch at the Endocrine Core Lab at Yerkes National Primate Research Center, Emory University, all in triplicate, using a modified kit from Diagnostic Systems Laboratories (Webster, TX). The sensitivity was $2-500 \mathrm{pg} / \mathrm{mL}$ per $200 \mu \mathrm{L}$ dose, and the interassay coefficient of variation was $8.77 \%$ at $0.65 \mathrm{ng} / \mathrm{mL}$ and $6.88 \%$ at $5.06 \mathrm{ng} / \mathrm{mL}$. The intra-assay coefficient of variation was $6.54 \%$ at $98.82 \mathrm{pg} / \mathrm{mL}$.

\section{Results}

Analyses were conducted with the Statistical Package for the Social Sciences (SPSS), v. 13.0. Group differences were analyzed with analyses of covariance (ANCOVA) separately for sex. Main effects of relationship type were evaluated with the LSD (Least Significant Difference) test after significant omnibus analyses. Covariates for analyses with $\mathrm{T}$ included age, sampling time, and sampling month, since all are associated with $\mathrm{T}$ and may be confounded with relationship status as per testing times. Correlations were conducted with Pearson Product Moment Correlations and partialled the effects of age. We included poly lifestyle women $(n=4)$ and men $(n=6)$ in analyses for exploratory purposes since we did not have specific relevant hypotheses but they constituted a group of interest for future study.

\section{Relationship type and testosterone}

\section{Men}

To see if men's T differed as a function of relationship type, we conducted a univariate ANCOVA. There was a significant overall effect, $F(3,37)=4.33, p=0.010$, partial $\eta^{2}=0.260$ (see Fig. 1a). Partnered men had significantly lower $\mathrm{T}$ than single men, $p=0.033$, polyamorous men, $p=0.001$, and poly lifestyle men, $p=0.005$. There was a trend for polyamorous men to have significantly higher $\mathrm{T}$ than single men, $p=0.073$. Controlling for BMI (body mass index: a measure of weight corrected for height) only increased the effect size (partial $\eta^{2}=0.293$ ) and resulted in the same pattern of significant results. Controlling for sexual orientation did not change the pattern of significant results. The association between $\mathrm{T}$ and relationship type was not dependent on SOI, since controlling for SOI did not change the pattern of significant results.

\section{Women}

There was one outlier who exhibited a $\mathrm{T}$ value that was over eight standard deviations away from the mean, which may have reflected blood contamination of the saliva sample; this participant was excluded from the endocrine assays and this reduced the number of partnered women to five. To see if women's $\mathrm{T}$ differed as a function of relationship type, we conducted a univariate ANCOVA. There was a significant overall effect, $F(3,32)=3.83, p=0.019$, partial $\eta^{2}=0.264$ (please see Fig. 1b). Polyamorous women had significantly higher $\mathrm{T}$ than single women, $p=0.020$, partnered women, $p=0.020$, and poly lifestyle women, $p=0.023$. Controlling for
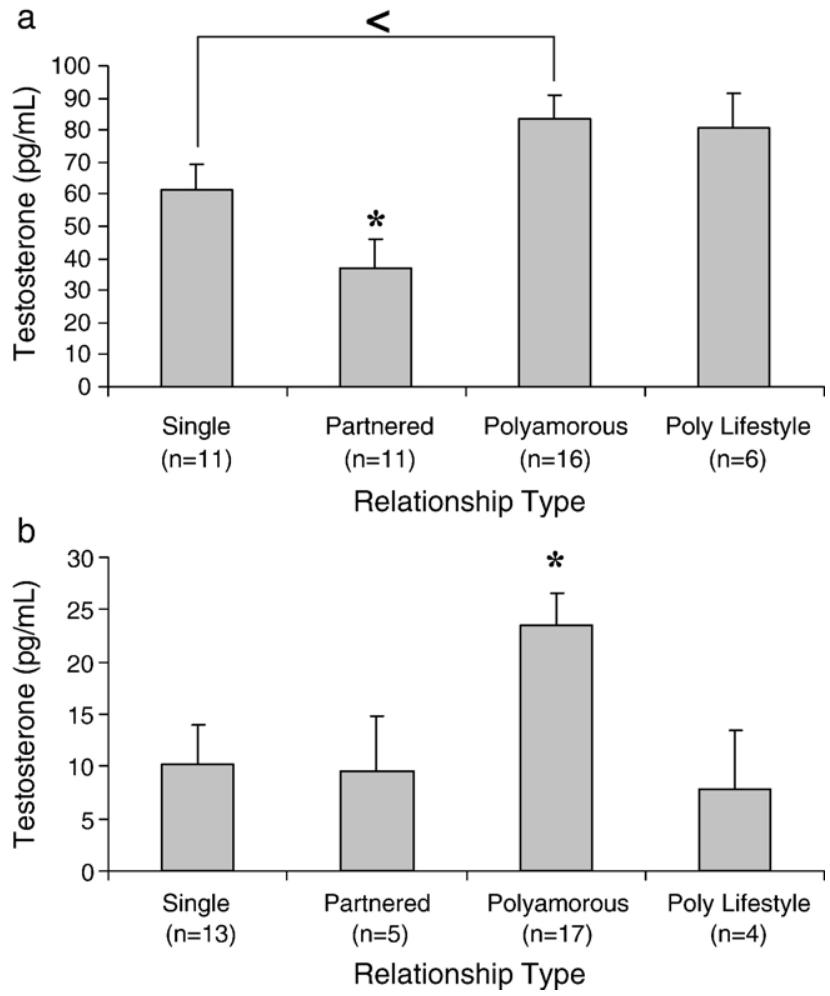

Fig. 1. Mean testosterone levels and standard errors by relationship type, adjusted for age, sampling month, and sampling time for (a) men and (b) women. 'Poly lifestyle' indicates participants not currently with multiple partners but identifying as having a poly approach to relationships. '*' indicates a significant difference from all other means at $p<0.05$. ' $<$ ' indicates a trend towards a significant difference from other means at $p<0.10$.

BMI or sexual orientation did not change the overall pattern of significant results. As in men, the association between $\mathrm{T}$ and relationship type was not dependent on SOI, since controlling for SOI did not change the pattern of significant results.

\section{Sociosexual orientation scores and sexual desire by relationship type}

Polyamory relates to multiple relationships, but also commitment within these, so it is unclear whether SOI scores should differ between polyamorous and partnered individuals. We conducted an independent $t$-test to see if SOI scores differed as a function of relationship type (partnered vs. polyamorous) (see Fig. 2). Polyamorous women did have significantly less restricted SOI scores than partnered women, $t(17)=-3.31$, $p=0.004$. We excluded one man from the polyamory group who had an SOI score over 17 standard deviations away from the mean. There was no significant difference in SOI scores between partnered and polyamorous men, $t(19)=0.121, p=0.732$.

To determine whether sexual desire differed by relationship type, we conducted separate ANCOVAs by sex with the three SDI measures (solitary, dyadic, total) as the dependent measures and relationship status as the independent measure, and age as the covariate (because sexual desire tends to decrease with age). In men, there were no significant overall effects of relationship 


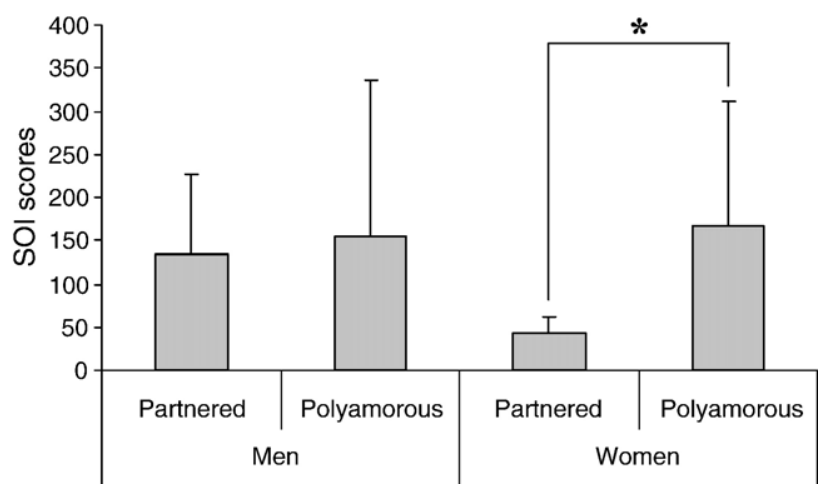

Fig. 2. Mean SOI scores (sociosexual orientation inventory) and standard deviations by sex and relationship type. '*' indicates a significant difference from all other means at $p<0.05$. Higher scores represent a less restricted sociosexual orientation.

type on solitary SDI, $F(3,35)=0.62, p=0.606$, dyadic SDI, $F(3,35)=0.20, p=0.897$, or total SDI, $F(3,35)=0.10, p=0.99$.

For women, however, there were significant overall effects for solitary SDI, $F(3,35)=4.24, p=0.012$, dyadic SDI, $F(3,35)=$ $2.89, p=0.049$, and total SDI, $F(3,35)=4.19, p=0.012$. Post hoc analyses (see Fig. 3) revealed that polyamorous women had significantly higher solitary SDI than single women, $p=0.040$, partnered women, $p=0.002$, and nearly so for poly lifestyle women, $p=0.058$. Similarly, polyamorous women had significantly higher dyadic SDI than single women, $p=0.007$, and nearly so compared to partnered women, $p=$ 0.092. Also similarly, polyamorous women had significantly higher total SDI than single women, $p=0.004$, partnered women, $p=0.014$, and nearly so for poly lifestyle women, $p=0.073$.

Thus, polyamorous women had significantly less restricted SOI scores than partnered women. And, polyamorous women had significantly higher sexual desire than other women. These differences were not apparent in men.

\section{Interrelations among SOI, sexual desire, and testosterone}

All partial correlations control for age.

\section{Men}

SOI was not significantly correlated with SDI: solitary SDI, partial $r(31)=-0.16, p=0.362$, dyadic SDI, $r(31)=-0.16$, $p=0.362$, or total SDI, $r(31)=0.06, p=0.740$.

SOI and $\mathrm{T}$ were not significantly correlated, partial $r(31)=$ $0.08, p=0.631$. There were no significant correlations between $\mathrm{T}$ and any of the individual SOI questions.

$\mathrm{T}$ was not significantly correlated with SDI scores with age controlled: solitary SDI, partial $r(35)=0.07, p=0.677$, dyadic SDI, partial $r(35)=-0.05, p=0.753$, total SDI, partial $r(35)=$ $-0.01, p=0.933$.

\section{Women}

SOI was not significantly correlated with SDI scores: solitary SDI, partial $r(33)=0.24, p=0.146$, dyadic SDI, partial $r(33)=$ $0.20, p=0.235$, or total SDI, partial $r(32)=0.23, p=0.172$.
SOI and $\mathrm{T}$ were not significantly correlated, partial $r(29)=$ $-0.04, p=0.840$. However, $\mathrm{T}$ was significantly correlated with two of the behavioral SOI questions: number of partners in the previous year, partial $r(31)=0.44, p=0.008$, and number of different sexual partners expected for the next 5 years, partial $r(30)=0.45, p=0.007$. This is likely explained by the association between multiple partners and higher $\mathrm{T}$.

$\mathrm{T}$ was not significantly correlated with dyadic SDI, partial $r(31)=0.22, p=0.200$, or total SDI, partial $r(30)=0.27$, $p=0.130$, but there was a trend for solitary SDI, partial $r(31)=0.33, p=0.053$.

\section{Discussion}

The present study examined testosterone (T) levels in women and men who were single, in monoamorous relationships (partnered), in polyamorous relationships, or in a polyamorous lifestyle. Our results showed that $\mathrm{T}$ was lower in partnered men than in single, polyamorous, or poly lifestyle men. This replicates previous findings showing that $\mathrm{T}$ is higher in unpartnered than partnered men (e.g. Booth and Dabbs, 1993; Mazur and Michalek, 1998; Gray et al., 2002; van Anders and Watson, 2006a). Our study is the first to report that $\mathrm{T}$ is higher in polyamorous than partnered men, supporting our hypothesis. Presumably, polyamorous individuals have a higher likelihood of additional partners (as this is explicitly part of their approach to relationships), which fits a competitive-type situation and should be associated with higher $\mathrm{T}$ under the testosterone tradeoff framework (van Anders and Watson, 2006b). We also found a trend for polyamorous men to exhibit higher $\mathrm{T}$ than single men. This supports (partially) our hypothesis that polyamorous men would display higher $\mathrm{T}$ than single men because polyamory is explicitly oriented toward the likelihood of new partners in a way that being single is not.

Our men's data suggest that having a partner, per se, is not associated with lower $\mathrm{T}$, since polyamorous men who had multiple partners exhibited higher $\mathrm{T}$ than monoamorously partnered men. This is similar to Gray (2003), where polygynously married men had higher $\mathrm{T}$ than monogamously

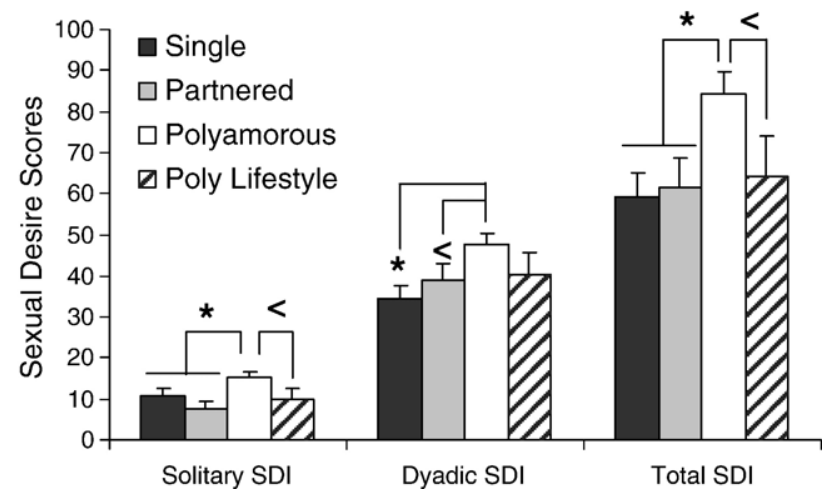

Fig. 3. Women's mean sexual desire and standard errors by relationship type, as measured on the Sexual Desire Inventory (SDI; Spector et al., 1996). '*' indicates a significant difference from all other means at $p<0.05$. ' $<$ ' indicates a trend towards a significant difference from other means at $p<0.10$. 
married men. Though our sample sizes were comparable to similar studies, replication with larger $n$ 's is still warranted. Still, previous studies have shown that higher $\mathrm{T}$ is associated with a competitive relationship orientation (propensity to enter shorter-term/sexual relationships), including more sexual partners (Bogaert and Fisher, 1995; Cashdan, 1995), more extramarital sex (Booth and Dabbs, 1993), less need for long-term commitment (Cashdan, 1995), and less relationship commitment (in partnered men; McIntyre et al., 2006). In addition, longitudinal studies have shown that $\mathrm{T}$ is higher in consistently single men and in men who divorce than in consistently wed men (Mazur and Michalek, 1998), and that higher T predicts staying unpartnered (van Anders and Watson, 2006a).

Our findings suggest that the T-partnering link in men is not driven by sexual desire or SOI, which did not differ significantly by relationship type, or even sexual activity, which likely did differ between single and polyamorous men. SOI, it should be noted, is a measure of willingness to engage in sexual activities outside of an exclusive pair bond, and as such does not address multiple partners of a sexual and romantic nature. An interesting aspect of polyamory is its focus on emotional commitment with multiple partners. Thus, men who were mono- or polyamorously partnered may have been similarly oriented toward bond-maintenance behaviors. It would thus be interesting to include men in multiple but not committed relationships in the future.

Our results from women show that polyamorous women have higher $\mathrm{T}$ than other women who are single, partnered, or in a poly lifestyle. These results support our first hypothesis that polyamorous women would have higher $\mathrm{T}$ than partnered women and support our second hypothesis that polyamorous women would have higher $\mathrm{T}$ than single women. These hypotheses, again, were based on the notion that polyamory would be a competitive-type context and thus associated with higher $\mathrm{T}$ in the testosterone trade-off framework (van Anders and Watson, 2006b). There was an implicit hypothesis, however, that was unsupported: that single women would have higher $\mathrm{T}$ than partnered women. This too was based on the testosterone tradeoff framework, as well as past research. These results should be cautiously interpreted in light of the small sample of partnered women, and further replication is warranted. One previous study has found that partnered women have lower $\mathrm{T}$ than unpartnered women, though this only reached significance in non-heterosexual women (van Anders and Watson, 2006a). The only other study that has examined partnering and $\mathrm{T}$ in women found that physical partner presence is associated with lower $\mathrm{T}$ (van Anders and Watson, 2007). Similar to the present study, these results were still apparent when analyses controlled for sexual orientation. Thus, though the findings from women in the present study confirmed our explicit hypotheses, they are not entirely consistent with our expectations.

The finding of higher sexual desire and SOI scores in polyamorous women may reflect trait and/or state associations. Accordingly, women may be more likely to be polyamorous because of higher sexual desire (or less restricted sociosexuality), or having more partners may increase women's sexual desire (or sociosexuality). Our study did not have a longitudinal component, so we cannot address the former. However, the finding that polyamorous women had a trend towards higher sexual desire than poly lifestyle women supports the latter. Since women who were oriented towards multiple partners (i.e. poly lifestyle) had lower sexual desire than women who currently had multiple partners (i.e. polyamorous women), the interpretation that multiple partners might increase sexual desire could be supported. Would imagining other sexual partners be similarly associated with higher sexual desire? If so, would there be a causal direction to this? The association between multiple partners and higher sexual desire is interesting and, with additional research, may prove to be informative and useful to researchers in the field of sexual desire (including low sexual desire).

Replications and extensions with both men and women are warranted because of both the smaller sample sizes (especially in monoamorously partnered women) and recruitment. The majority of the polyamorous individuals were recruited from polyamory group meetings or listservs with a minority through advertisements, whereas the majority of non-polyamorous participants were recruited through advertisements or general listservs. It is possible, then, that the polyamorous participants, who belonged to these groups, may have been more generally social than the non-polyamorous participants.

The data are beginning to consistently point to an association between relationship orientation and $\mathrm{T}$ in men, but the association between $\mathrm{T}$ and partnering in women appears to be either more complex or less immediately apparent. In women, data support both a state interpretation (i.e. that relationship status is associated with $\mathrm{T}$ ), and a trait interpretation (i.e. that $\mathrm{T}$ predicts partnering). Still, lower $\mathrm{T}$ is at least consistently found in women who are in relationships with one person, though this is not always the exclusive case and there appear to be inconsistent moderators of this association (e.g. sexual orientation). Only further data will clarify the underlying pattern in women and will be valuable in our understanding of how androgens and partnering are associated.

\section{Acknowledgments}

This research was supported by Discovery Grant 0194522 from the Natural Sciences and Engineering Research Council of Canada (NSERC) to N.V. Watson. S.M. van Anders was supported by a War Memorial Scholarship from IODE Canada. Salivary assays were conducted by the Endocrine Core Assay $\mathrm{Lab}$ at Yerkes Primate Research Center, Emory University. We would like to thank N. Macdonald, S. Orford, G. Panduri, R. Park, N. Schmidt, C. Viray, and E. Wagner for help with data collection. We would also like to thank B.C. Jones and two anonymous reviewers for comments on previous drafts. L.D. Hamilton is now a Ph.D. student in the Department of Psychology at the University of Texas, Austin.

\section{References}

Booth, A., Dabbs Jr., J.M., 1993. Testosterone and men's marriages. Soc. Forces $72,463-477$. 
Bogaert, A.F., Fisher, W.A., 1995. Predictors of university men's number of sexual partners. J. Sex Res. 32, 119-130.

Burnham, T.C., Flynn Chapman, J., Gray, P.B., McIntyre, M.H., Lipson, S.F., Ellison, P.T., 2003. Men in committed romantic relationships have lower testosterone. Horm. Behav. 44, 119-122.

Cashdan, E., 1995. Hormones, sex, and status in women. Horm. Behav. 29 (3), 354-366.

Dabbs Jr., J.M., de la Rue, D., 1990. Salivary testosterone measurements among women: relative magnitude of circadian and menstrual cycles. Horm. Res. 35 (5), 182-184.

Gangestad, S.W., Simpson, J.A., Cousins, A.J., Garver-Apgar, C.E., Christensen, P.N., 2004. Women's preferences for male behavioral displays change across the menstrual cycle. Psychol. Sci 15, 203-207.

Gray, P.B., 2003. Marriage, parenting, and testosterone variation among Kenyan Swahili men. Am. J. Phys. Anthropol. 122 (3), 279-286.

Gray, P.B., Kahlenberg, S.M., Barrett, E.S., Lipson, S.F., Ellison, P.T., 2002. Marriage and fatherhood are associated with lower testosterone in males. Evol. Hum. Behav. 23, 193-201.

Gray, P.B., Campbell, B.C., Marlowe, F.W., Lipson, S.F., Ellison, P.T., 2004a. Social variables predict between-subject but not day-to-day variation in the testosterone of US men. Psychoneuroendocrinology 29, 1153-1162.

Gray, P.B., Flynn Chapman, J., Burnham, T.C., McIntyre, M.H., Lipson, S.F., Ellison, P.T., 2004b. Human male pair bonding and testosterone. Hum. Nat. $15,119-131$.

Introduction to Polyamory, n.d. Retrieved November 15th, 2006, from http:/ www.polyamorysociety.org/page6.html.

Jones, B.C., Little, A.C., Boothroyd, L., Debruine, L.M., Feinberg, D.R., Smith, M.J., Cornwall, R.E., Moore, F.R., Perrett, D.I., 2005. Commitment to relationships and preferences for femininity and apparent health in faces are strongest on days of the menstrual cycle when progesterone level is high. Horm. Behav. 48, 283-290.

Kinsey, A.C., Pomeroy, W.B., Martin, C.E., Gebhard, P.H., 1948. Sexual Behavior in the Human Male. WB Saunders, Philadelphia.

Mazur, A., Michalek, J., 1998. Marriage, divorce, and male testosterone. Soc. Forces 77 (1), 315-330.

McIntyre, M.H., Gangestad, S.W., Gray, P.B., Chapman, J.F., Burnham, T.C., O’Rourke, M.T., Thornhill, R., 2006. Romantic involvement often reduces men's testosterone levels - But not always: the moderating role of extrapair sexual interest. J. Pers. Soc. Psychol. 91 (4), 642-651.

Sakaguchi, K., Oki, M., Honma, S., Hasegawa, T., 2006. Influence of relationship status and personality traits on salivary testosterone in Japanese men. Pers. Individ. Differ. 41, 1077-1087.

Simpson, J.A., Gangestad, S.W., 1991. Individual differences in sociosexuality. J. Pers. Soc. Psychol. 60 (6), 870-883.

Spector, I.P., Carey, M.P., Steinberg, L., 1996. The Sexual Desire Inventory: development, factor structure, and evidence of reliability. J. Sex Marital Ther. 22, 175-190.

van Anders, S.M., Watson, N.V., 2006a. Relationship status and testosterone in North American men and women of diverse orientations: cross-sectional and longitudinal findings. Psychoneuroendocrinology 31, 715-723.

van Anders, S.M., Watson, N.V., 2006b. Social neuroendocrinology: effects of social behaviors and contexts on sex steroids in humans. Hum. Nat. 17 (2), 212-237.

van Anders, S.M., Watson, N.V., 2007. Testosterone levels in women and men who are single, in long-distance relationships, or same-city relationships. Horm. Behav. 51, 286-291.

van Anders, S.M., Hampson, E., Watson, N.V., 2006. Seasonality, waist-to-hip ratio, and salivary testosterone. Psychoneuroendocrinology 31 (7), 895-899. 